\title{
The Significance of Electrocardiographic Abnormalities and Serology for
} T. cruzi Infection

Monique Lirio', Zuinara Pereira Gusmão Maia', Maria Nakatani', Sanjay R. Mehta² and Roberto Badaro ${ }^{1 *}$
${ }^{1}$ Federal University of Bahia Hospital Complex, Bahia, Brazil

${ }^{2}$ Division of Infectious Diseases, University of California San Diego, California, USA

\begin{abstract}
Background: Electrocardiography (EKG) is a basic complementary exam in the evaluation patients with Chagas disease (CD), where findings can precede symptoms and physical examination abnormalities.

Objective: To correlate positive Chagas serology and electrocardiographic abnormalities associated with chronic chagasic cardiomyopathy.

Method: We evaluated the correlation between results of an ELISA using crude antigen (TcLys) and recombinant antigen (TcF) with the presence of EKG disturbances, within a cohort of individuals with either epidemiologic risk or clinical symptoms suggestive of CD, sent to our Laboratory for Tropical Diseases for testing in state of Bahia, Brazil.

Results: 84 individuals had a positive ELISA using TcLys or TcF antigen. Overall, 49 patients (58.3\%) were symptomatic with CD, $42(85.7 \%)$ with isolated evidence of the cardiac form, one $(2.0 \%)$ with megacolon and mixed (megaviscera and cardiac) in six (12.2\%). TcLys ELISA was positive in 45/49 (91.8\%) and TcF in 42/49 (85.7\%) of the symptomatic patients. The most common EKG abnormality, Complete Right Bundle Branch Block (CRBBB), was seen in 47/84 patients (56.0\%) of the patients. Interestingly, in $11 / 47(23.4 \%)$ of the patients with CRBBB, serologies were discordant. Conclusions: EKG plays a key role in the initial evaluation of patients with positive Chagas serology using crude or recombinant antigens. Individuals with positive serology should be carefully followed with periodical cardiac medical examination to early detect EKG abnormality compatible with CD.
\end{abstract}

Keywords: Chagas' disease; Serology; Electrocardiography

\section{Introduction}

Chagas Disease (CD), also referred to as American trypanosomiasis, remains a major Latin America public health problem. The estimated prevalence of CD in the Americas is between 18 - 24.7 million cases [1]. $\mathrm{CD}$ is caused by the infection of the protozoan parasite Trypanosoma cruzi, naturally transmitted by the triatomine reduviid bugs [2]. Blood transfusions are also responsible for a significant number of infected patients [3]. Recently, accidental ingestion of sugarcane and açai fruit juice contaminated with T. cruzi from infected triatomines crushed during the preparation of the juice has been associated with an outbreak of acute Chagas' disease [4-6]. The disease occurs in two stages. The acute phase occurs is typically either asymptomatic, or associated with fever and other mild symptoms. One of the classic manifestations of acute CD is Romaña's sign, which is swelling of the eye on one side of the face, usually at the bite site where insect feces was rubbed into the eye. The other nonspecific symptoms seen in acute CD include fatigue, fever, an enlarged liver or spleen, and swollen lymph glands. Rarely, rash, loss of appetite, diarrhea, and vomiting can occur. In general, symptoms last for 4-8 weeks and then resolve, even without treatment $[7,8]$. Nevertheless, occasionally, life-threatening myocarditis or meningoencephalitis can occur during the acute phase, particularly in young children and immunocompromised persons $[7,9]$.

The majority of infected individuals remain asymptomatic, but an unknown number of initially asymptomatic individuals eventually develop symptoms during the chronic phase of disease after a silent period that may last several years. The pathology of chronic Chagas'disease can result in irreversible changes to the heart, the esophagus and the colon [10], and also may affect the peripheral nervous system [11]. An estimated 30\% of those infected with CD develop cardiac symptoms which may lead to sudden death, while approximately $6 \%$ develop gastrointestinal pathology such as megaviscera $[8,10,12]$ and at least $3 \%$ present with peripheral nervous involvement [11]. In patients who are immunocompromised, including persons with HIV/AIDS, Chagas disease can be fatal. In these patients, difficult to treat unifocal or multifocal chagomas (parastic lesions) may develop in the brain, resulting in menigoencephalitis in $70 \%$ of these cases [13]. Even individuals who remain asymptomatic probably are infected and infectious for life, with low levels of the parasite in their blood and other tissues.

Cardiac involvement is characterized by an inflammatory and fibrosing cardiomyopathy with progressive failure of myocardial contractile function. Parasite persistence and the parasite-driven immune response are considered the main mechanisms that explain the pathogenesis of Chagas heart disease [14]. Chronic Chagas cardiomyopathy presents as three major conditions: cardiac dysrhythmia, heart failure and thromboembolism, all of which may coexist in the same individual $[8,15]$. Clinical findings vary extensively according to disease duration and the extent and site of cardiac lesions. Hence, some individuals may have a form of Chagas disease with preserved ventricular function, characterized only by arrhythmias and intraventricular or atrioventricular conduction disturbances $[12,16]$. It has been well established in the natural history of CD that Electrocardiography (EKG) disturbances precede symptoms and

*Corresponding author: Roberto Badaro, Federal University of Bahia Hospital Complex, Bahia, Brazil, Tel: (55 71) 3235-4901/3283-6207; Fax: (55 71) 32472756; E-mail: rbadaro884@gmail.com; badaro@ufba.br

Received October 09, 2013; Accepted October 28, 2013; Published October 30 , 2013

Citation: Lirio M, Maia ZPG, Nakatani M, Mehta SR, Badaro R, et al. (2013) The Significance of Electrocardiographic Abnormalities and Serology for T. cruzi Infection. J Trop Dis 1: 121. doi: 10.4172/2329-891X.1000121

Copyright: (C) 2013 Lirio M, et al. This is an open-access article distributed unde the terms of the Creative Commons Attribution License, which permits unrestricted use, distribution, and reproduction in any medium, provided the original author and source are credited. 
physical examination findings [12]. Right Bundle Branch Block (RBBB) is the most common EKG finding seen in CD, and thus may act as a predictor of more serious disease. Nevertheless, it remains a challenge in the management of $\mathrm{CD}$ to predict which individuals are at risk of developing disturbances associated with chronic forms of the disease. It is not well established whether positive serology or level of serum antibodies are related to the development of cardiac or digestive abnormalities.

Due to the low parasitemia during the chronic stage of the disease, parasitological tests are not routinely used. Therefore, serological tests based on the detection of antibodies to T. cruzi are the most useful diagnostic tool [17]. Most commercially available Enzyme Linked Immunosorbent Assays (ELISA) use antigens obtained from lysis of the epimastigote or trypomastigote forms of the parasite (TcLys). These tests have high sensitivity, but often fail to distinguish which antibodies are specific for T. cruzi $[18,19]$. In recent years, several recombinant antigens and synthetic peptides have been developed for the serologic diagnosis of $\mathrm{CD}[20-22]$. TcF is a recombinant antigen composed of four antigens developed from the combination TcLo1.2 and the tripeptide $2 / \mathrm{D} / \mathrm{E}$, which consists of the peptides PEP-2, TcD and TcE. This polypeptide was previously studied in a group of patients with confirmed Chagas' disease from endemic areas in Brazil [23]. The polypeptide based ELISA demonstrated a high sensitivity and specificity when compared with indirect immunofluorescence tests and ELISA using crude lysate antigens [23,24].

The aim of this study was to determine the correlation between the results of ELISAs using crude antigen (TcLys) and recombinant antigen (TcF) with EKG abnormalities associated with chronic chagasic cardiomyopathy in a group of individuals from an endemic area of T. cruzi infection in Brazil.

\section{Materials and Methods}

\section{Study design and sample definition}

We conducted a retrospective cross-sectional study to identify electrocardiographic abnormalities associated with of Chagas' disease in individuals that had performed ELISAs using T. cruzi crude and recombinant antigens. This study was carried out at the main University Hospital in Salvador, Brazil, Complexo Hospitalar Universitário Professor Edgard Santos (C-HUPES). This study was approved by the Ethical Research Committee of the Complexo Hospitalar Universitário Professor Edgard Santos under number 097/09.

The study population was comprised of individuals who were referred to the Laboratory of Tropical Diseases at C-HUPES for clinical findings or epidemiology that suggested Chagas' disease. These individuals were tested with both the standard of care TcLys-ELISA and the experimental TcF-ELISA during a period extending from June 2006 to October 2008.

\section{Clinical data}

For all individuals included in the study we collected complementary exams, clinical and demographic data from medical charts. EKG and barium enema radiographic studies were performed in the cardiology and gastroenterology department of C-HUPES, respectively. EKG abnormalities including: complete right bundle branch block (with or without left anterior fascicular block), left bundle branch block, 2nd and 3rd degree intraventricular conduction blocks, sinus node dysfunction, atrial fibrillation, ventricular extra systoles, electrically inactive area, primary $\mathrm{T}$ wave abnormalities, and heart rate under $40 \mathrm{bpm}$ were considered suggestive of Chagas cardiomyopathy, based on the Brazilian Consensus Statement on Chagas disease [10]. Other findings were considered as unspecific isolated abnormalities. Barium studies were used to identify the presence of megaesophagus and megacolon. In the absence of abnormal findings on these tests, individuals were classified as possible carriers of the indeterminate form of $\mathrm{CD}$. The presence of other comorbidities was also evaluated.

\section{ELISA testing}

Antigen source: Epimastigotes forms of Trypanosome cruzi were obtained and lysate (TcLys) following a previous established protocol described elsewhere [23].

Microtiter plates (Nunc ImmunoModule Plates, maxiSorp surface, no. 473768 , Roskilde, Denmark) were coated using $50 \mu \mathrm{l} /$ well of the TcLys $(1 \mu \mathrm{g} /$ well $)$ or TcF, (200 ng/well) antigens diluted in carbonatebicarbonate buffer $\mathrm{pH} 9.6,37^{\circ} \mathrm{C}$ and incubated overnight at $7^{\circ} \mathrm{C}$. For plate blocking, $200 \mu \mathrm{l}$ of blocking buffer (PBS 1X+1\% skimmed milk powder was used) was added to each well and incubated for 2 hours, followed by five washes with wash buffer. Human sera were then diluted at a ratio of 1:100 in PBS+0.1\% BSA $+0.05 \%$ Tween 20 and distributed in $50 \mu \mathrm{l} /$ well and incubated for at least 20 minutes at room temperature. Negative controls and serum of individuals were tested at minimum in duplicate. The plate was washed five times with washing buffer (PBS-T) and then peroxidase conjugate Protein A from Staphylococcus aureus - total IgG (Sigma P 8651, St. Louis, USA) diluted at a ratio of 1:20,000 in $1 \mathrm{X}$ PBS $+0.1 \% \mathrm{BSA}+0.05 \%$ Tween 20 was added and the plates were then incubated for 20 minutes at room temperature. The plate was again washed five times with PBS-T and $50 \mu \mathrm{l} /$ well of substrate enzyme 3,3; 5,5-TetraMethylbenzidine (TMB) produced by Kierkegaard \& Perry Laboratories Inc. Gaithersburg - USA, was added and the plate incubated for 15 minutes. Finally, $50 \mu \mathrm{l} /$ well of $1 \mathrm{~N} \mathrm{H}_{2} \mathrm{SO}_{4}$ was added to stop the color reaction. The micro plate was read in spectrophotometer at $450 \mathrm{~nm}$ filter.

\section{Data analysis}

The cut off value for a positive test was derived from the average of three optical densities from the negative controls plus three standard deviations. Negative controls were obtained from the serum bank of the Laboratory of Tropical Diseases and included healthy individuals with no clinical signs and negative epidemiology for Chagas' disease. For each ELISA experiment, a negative control in duplicate was used. Based on these results, an optical density greater than or equal to 1.4 was considered positive.

\section{Results}

We reviewed ELISA results and clinical data from the charts of 161 patients with epidemiology or clinical symptoms suggestive of Chagas' disease. A total of 77 patients were excluded due to due to missing clinical or complementary exams data in medical charts. The final study population included 84 individuals with positive serology for T. cruzi infection using either the TcLys or TcF antigens. The study population included 31 men and 53 women ranging in age from 21-85 years (mean $51.6 \pm 13.7$ ). Table 1 presents the frequency of EKG changes and other comorbidity in the studied population. As noted, the majority 47/84 (56\%) had the most common EKG abnormality seen in patients with Chagas' disease, Complete Right Bundle Branch Block (CRBBB).

Forty-nine out of the 84 patients (58.3\%) had a clinical manifestation compatible with one of the clinical forms of $\mathrm{CD}$. Among them, the cardiac form was suspected in $48 / 49$ (98\%) including 47 with CRBBB 
Citation: Lirio M, Maia ZPG, Nakatani M, Mehta SR, Badaro R, et al. (2013) The Significance of Electrocardiographic Abnormalities and Serology for T. cruzi Infection. J Trop Dis 1: 121. doi: 10.4172/2329-891X.1000121

and one with isolated intraventricular block. Both the classic cardiac form, cordis bovis, (Figure 1) and megacolon were seen (Figure 2). No subjects had megaesophagus, (Figure 3). TcLys ELISA was positive in 38 (90.5\%) and $\mathrm{TcF}$ in 35 (85.3\%) of the patients with EKG abnormalities compatible with $\mathrm{CD}$ as shown in Table 2. Interestingly, in eleven patients in the group with suspected cardiac Chagas' disease, discordant serology results were documented between the TcLys and TcF antigens. Table 3 depicts the EKG type of abnormalities in each of these patients. Only one did not have CRBBB, but had Intraventricular Block (IVB), also compatible with CD. The other 35 patients in the studied population with serology positive either TcLys or TcF were asymptomatic, had a normal EKG and were considered to have an indeterminate form of $\mathrm{CD}$.

\begin{tabular}{|l|c|c|}
\hline Condition & $\mathbf{n}$ & $\%$ \\
\hline Abnormality & & \\
\hline Complete right bundle branch block & 47 & 56 \\
\hline Intraventricular conduction blocks & 08 & 2.8 \\
\hline Left bundle branch block & 02 & 1.1 \\
\hline Ventricular extra systoles & 01 & 1.1 \\
\hline Electrically inactive area & 01 & 36.6 \\
\hline Unspecific isolated abnormalities & 33 & \\
\hline Comorbidity* & & 41.1 \\
\hline Hypertension & 37 & 7.7 \\
\hline Type 2 diabetes & 07 & 5.5 \\
\hline Dyslipidemia & 05 & 3.3 \\
\hline Osteoarthritis & 03 & 2.2 \\
\hline Leishmaniasis & 02 & 1.1 \\
\hline HCV & 01 & 19.8 \\
\hline Other & 18 & \\
\hline
\end{tabular}

* Patients could present more than one EKG abnormality or comorbidity Table 1: Frequencies of EKG abnormalities and comorbidities in all 84 patients studied patients.

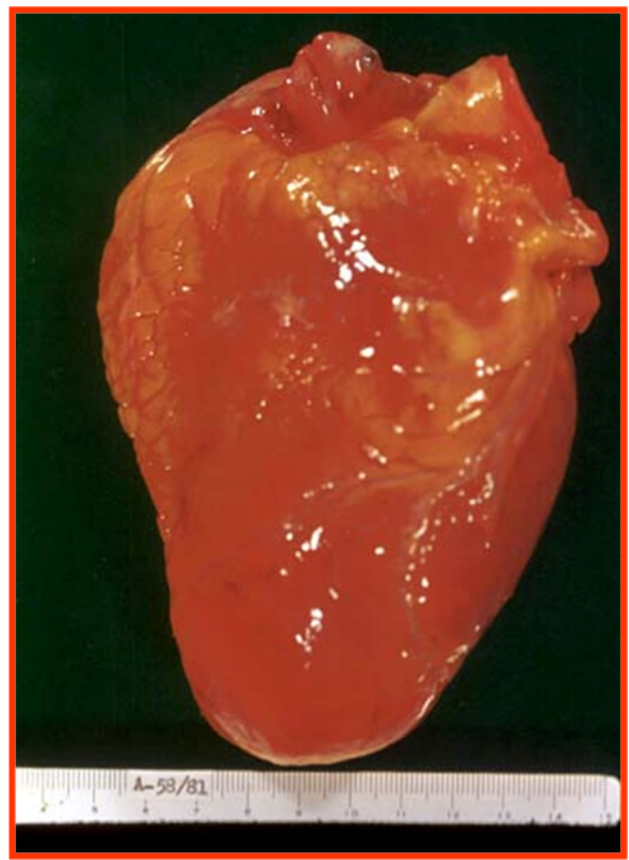

The aneurismatic feature of tip of an enlarged heart from an autopsy of a chagas disease patient

Figure 1: Typical cardiomegaly (cordis bovis) seen in a patient with Chagas' disease.
Results of TcLys and TcF ELISA were compared among the groups of individuals. The mean optical density divided by the cut off in TcLys ELISA was $4.4( \pm 3.0)$ in patients with the cardiac form, $3.4( \pm$ $2.5)$ in those with digestive or mixed form and $2.2( \pm 2.0)$ in those asymptomatic. In TcF ELISA, the groups with cardiac, digestive/mixed and asymptomatic forms presented mean of optical density / cut off of $3.3( \pm 2.1), 3.0( \pm 1.0)$ and $2.2( \pm 2.0)$, respectively. Figure 1 shows the range of results in both ELISA tests.

\section{Discussion}

EKG is a basic complementary exam in evaluation patients with Chagas disease and may be the only available diagnostic instrument in poverty-stricken areas, where there is often higher prevalence of T. cruzi infected patients. CRBBB is the most common EKG abnormality documented in patients with the cardiac form of Chagas' disease [12,25], and in our study, $56 \%$ of individuals had CRBBB isolated or combined
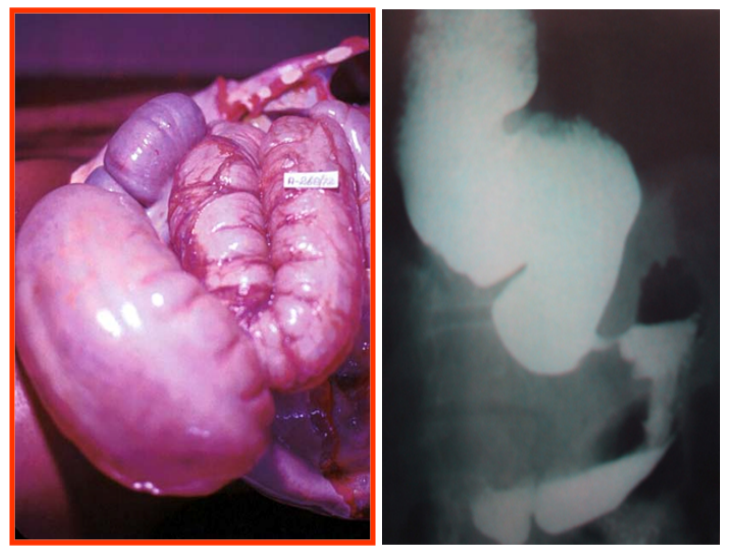

The left side is a megacolon feature of an autopsy of a patient with Chagas disease. On the right side is the radiographic barium enema characteristic of megacolon disease.

Figure 2: The typical megacolon documented in a patient with Chagas disease.
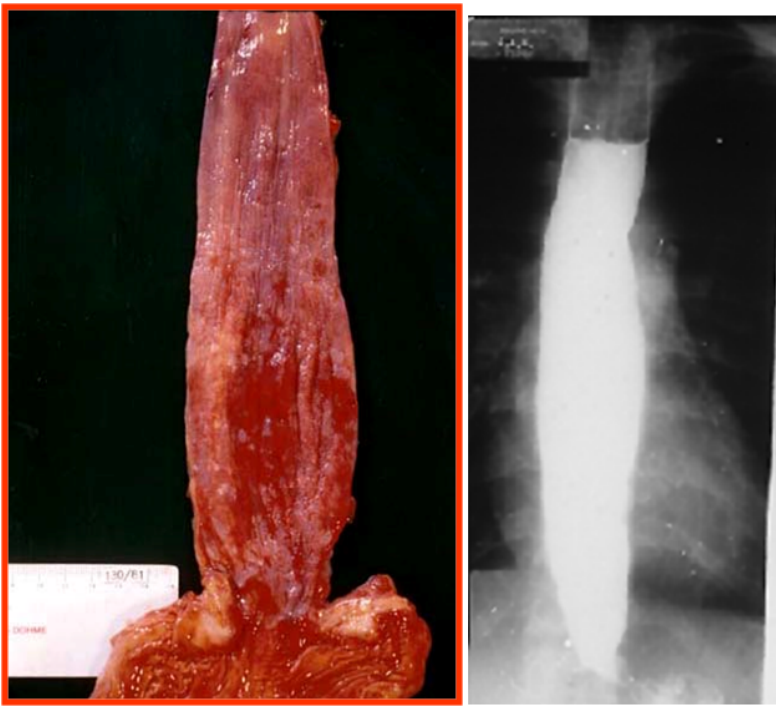

On the the left side, a megaesophagus is shown from an autopsy of a patient with $C D$. On the right side is the typical radiographic barium enema image

Figure 3: Megasophagus caused by Chagas' disease. 


\begin{tabular}{|l|c|c|c|c|}
\hline $\begin{array}{l}\text { Chagas Disease } \\
\text { Form }\end{array}$ & $\begin{array}{c}\text { Number of } \\
\text { patients }\end{array}$ & $\begin{array}{c}\text { TcLys ELISA } \\
\text { positive }\end{array}$ & $\begin{array}{c}\text { TcF ELISA } \\
\text { Positive }\end{array}$ & $\begin{array}{c}\text { TcLYS and TcF } \\
\text { ELISA positive }\end{array}$ \\
\hline Cardiac & 42 & 38 & 35 & 31 \\
\hline Megaesophagus & 0 & 0 & 0 & 0 \\
\hline Megacolon & 1 & 1 & 1 & 1 \\
\hline Mixed* & 6 & 6 & 6 & 6 \\
\hline Total & 49 & 45 & 42 & 39 \\
\hline
\end{tabular}

TcLys ELISA= indirect Enzyme immunosorbent assay with T. cruzi lysate antigen; TcF ELISA = indirect Enzyme immunosorbent assay with $T$. cruzi recombinan antigen; *Mixed forms: cardiac and megaesophagus 3 patients; cardiac and megacolon 2 patients; 1 patient with all three clinical CD forms

Table 2: Serologic tests to detect anti $T$. cruzi antibodies among patients with clinical forms of Chagas'Disease.

\begin{tabular}{|c|c|c|c|c|c|c|c|c|}
\hline Patient & Age (years) & CRBBB & IVB & EIA & LBBB & VES & TcLys & TcF \\
\hline$\# 1$ & 32 & Yes & yes & no & no & no & - & + \\
\hline$\# 2$ & 66 & No & yes & no & no & no & - & + \\
\hline$\# 3$ & 76 & Yes & No & no & no & no & - & + \\
\hline$\# 4$ & 40 & Yes & No & no & no & no & - & + \\
\hline$\# 5$ & 42 & Yes & No & no & no & no & + & - \\
\hline$\# 6$ & 57 & yes & No & no & no & no & + & - \\
\hline$\# 7$ & 67 & yes & No & no & no & no & + & - \\
\hline$\# 8$ & 55 & yes & No & no & no & no & + & - \\
\hline$\# 9$ & 52 & yes & No & no & no & no & + & - \\
\hline$\# 10$ & 39 & yes & yes & no & no & no & + & - \\
\hline$\# 11$ & 40 & yes & No & no & no & no & + & - \\
\hline
\end{tabular}

CRBBB: complete right bundle branch block; IVB: intraventricular conduction blocks; EIA: electrically inactive area; LBBB: left bundle branch block; VES ventricular extra systoles, TcLys ELISA= indirect Enzyme immunosorbent assay with $T$. cruzi lysate antigen; TcF ELISA= indirect Enzyme immunosorbent assay with $T$. cruzi recombinant antigen

Table 3: EKG characteristics of patients with TcLys Elisa or TcF Elisa negative.

with other EKG disturbances. Intraventricular conduction block was the second most frequent electrocardiographic abnormality seen.

Serologic testing is considered the most practical standard method to screen for T. cruzi infection [26]. In our study, both crude and recombinant antigens showed more than $85 \%$ of positivity among individuals with EKG abnormalities suggestible of CD. In the presence of digestive symptoms, the combined use of both ELISAs showed higher number of positive results. While it is not well established how well clinical signs and symptoms can be used to predict chronic forms of the disease, it has been shown that the clinical diagnosis of cardiac Chagas' disease and EKG abnormalities are associated with T. cruzi seropositivity [27]. Our results showed higher level of antibodies in individuals with the cardiac form of $\mathrm{CD}$ comparing to asymptomatic patients (Figure 4).

Many years ago, a prospective study of CD in a highly endemic area in Brazil revealed that $20.2 \%$ of individuals with $T$. cruzi infection had EKG abnormalities [25]. At that time only crude lysate antigen preparation was available for the ELISA. Our results are compatible with a more recent study on cardiac abnormalities in patients with T. cruzi infection, which have shown that EKG disturbances are present in $92 \%$ of seropositive individuals [28]. This suggests that in conjuction with a crude lysate ELISA, EKG may be a useful adjunctive tool in the diagnosis of CD.

Several studies have shown that recombinant antigens are more specific in the detection of anti-T. cruzi antibodies than crude antigen preparations, but may also be less sensitive [20,29]. Evaluations of
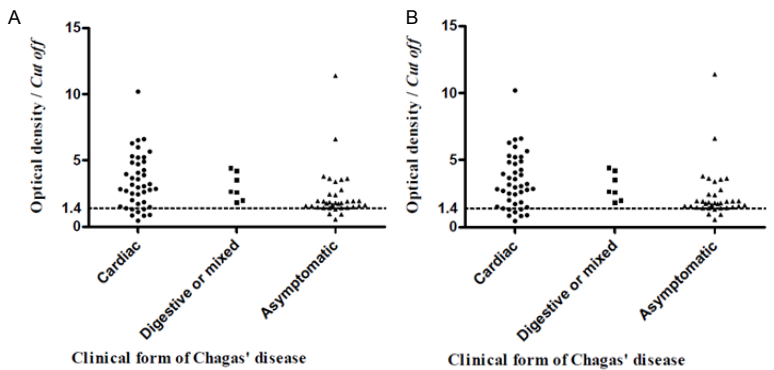

Dots are each value of optical density/cutoff from individuals with the cardiac form of CD. $v$ Squares are values of optical density/cutoff from individuals with megaviscera form of CD. $\Delta$ Triangles are values of optical density/cutoff from individuals with asymptomatic form of $C D$

Figure 4: Optical density / cut off results in TcLys (A) and TcF (B) ELISA according to Chagas' disease clinical form.

serodiagnostic tests using six different $T$. cruzi recombinant antigens have shown sensitivities varying from $4.2 \%$ to $97 \%$. However, the use of combinations of recombinant peptides has improved the sensitivity of serodiagnostic tests to nearly $100 \%$ [30]. Nevertheless, in our study we found no significant differences between the positive rates of TcLys compared to the recombinants peptides used in the ELISA.

WHO recommends the use of three different serological tests for confirm the T. cruzi positive serology and only consider individuals who have a test positive on two or more tests to be a true positive serology, due to the critical the poor specificity of many available commercial serological tests for CD [17]. However, it is difficult to have access to all these diagnostic tools in many endemic areas of CD. A combination approach using serology and EKG, may be an alternative method to obtain a more reliable diagnosis [26].

In conclusion, our results demonstrate that EKG can play a key role in the initial evaluation of patients with positive serology using crude or recombinant antigens. It has been shown that the relative risk of a person with positive serology for CD compared to a seronegative individual for ventricular dysfunctions is 3.69 , and if the person has any EKG abnormality at diagnosis, this risk is 2.72 -fold higher [12]. Thus, not only do we recommend the use of EKG in the initial evaluation of subjects for $\mathrm{CD}$, we also strongly recommend that individuals in endemic areas with serologic or EKG evidence of CD should be carefully followed with periodical cardiac medical examination, especially when a right bundle branch block is present.

\section{Acknowledgement}

We are indebt with Dr Steven G Reed from Infectious Disease Research Institute, Seattle, Washington, USA, who provided the recombinant T. cruzi antigens.

\section{References}

1. Schmunis GA, Yadon ZE (2010) Chagas disease: a Latin American health problem becoming a world health problem. Acta Trop 115: 14-21.

2. Araújo CA, Waniek PJ, Jansen AM (2009) An overview of Chagas disease and the role of triatomines on its distribution in Brazil. Vector Borne Zoonotic Dis 9: $227-234$.

3. Assal A, Corbi C (2011) [Chagas disease and blood transfusion: an emerging issue in non-endemic countries]. Transfus Clin Biol 18: 286-291.

4. de Souza-Lima Rde C, Vale Barbosa Md, Coura JR, Lima Arcanjo AR, da Silva Nascimento A, et al. (2013) Outbreak of acute Chagas disease associated with oral transmission in the Rio Negro region, Brazilian Amazon. Rev Soc Bras 
Citation: Lirio M, Maia ZPG, Nakatani M, Mehta SR, Badaro R, et al. (2013) The Significance of Electrocardiographic Abnormalities and Serology for T. cruzi Infection. J Trop Dis 1: 121. doi: 10.4172/2329-891X.1000121

Page 5 of 5

Med Trop 46: 510-514.

5. Dias JP, Bastos C, Araújo E, Mascarenhas AV, Martins Netto E, et al. (2008) Acute Chagas disease outbreak associated with oral transmission. Rev Soc Bras Med Trop 41: 296-300.

6. Nóbrega AA, Garcia MH, Tatto E, Obara MT, Costa E, et al. (2009) Oral transmission of Chagas disease by consumption of açaí palm fruit, Brazil. Emerg Infect Dis 15: 653-655

7. Bastos CJ, Aras R, Mota G, Reis F, Dias JP, et al. (2010) Clinical outcomes of thirteen patients with acute chagas disease acquired through oral transmission from two urban outbreaks in northeastern Brazil. PLoS Negl Trop Dis 4: e711.

8. Rassi Jr A, Rassi A, Marin-Neto JA (2009) Chagas heart disease: pathophysiologic mechanisms, prognostic factors and risk stratification. Mem Inst Oswaldo Cruz 104: 152-158.

9. Córdova E, Maiolo E, Corti M, Orduña T (2010) Neurological manifestations of Chagas' disease. Neurol Res 32: 238-244.

10. Roca C, Pinazo MJ, López-Chejade P, Bayó J, Posada E, et al. (2011) Chagas disease among the Latin American adult population attending in a primary care center in Barcelona, Spain. PLoS Negl Trop Dis 5: e1135.

11. Pittella JE (2009) Central nervous system involvement in Chagas disease: a hundred-year-old history. Trans R Soc Trop Med Hyg 103: 973-978.

12. Marques DS, Canesin MF, Barutta JF, Fuganti CJ, Barretto AC (2006) Evaluation of asymptomatic patients with chronic Chagas disease through ambulatory electrocardiogram, echocardiogram and B-Type natriuretic peptide analyses. Arq Bras Cardiol 87: 336-343.

13. Diazgranados CA, Saavedra-Trujillo $\mathrm{CH}$, Mantilla M, Valderrama SL, Alquichire C, et al. (2009) Chagasic encephalitis in HIV patients: common presentation of an evolving epidemiological and clinical association. Lancet Infect Dis 9: 324-330.

14. Bonney KM, Engman DM (2008) Chagas heart disease pathogenesis: one mechanism or many? Curr Mol Med 8: 510-518.

15. Nunes MC, Dones W, Morillo CA, Encina JJ, Ribeiro AL; Council on Chagas Disease of the Interamerican Society of Cardiology (2013) Chagas disease: an overview of clinical and epidemiological aspects. J Am Coll Cardiol 62: 767-776.

16. Acquatella $\mathrm{H}$ (2007) Echocardiography in Chagas heart disease. Circulation 115: $1124-1131$.

17. World Health Organization (2009) Neglected Tropical Diseases. Chagas disease (American trypanosomiasis).

18. Chiller TM, Samudio MA, Zoulek G (1990) IgG antibody reactivity with Trypanosoma cruzi and Leishmania antigens in sera of patients with Chagas' disease and leishmaniasis. Am J Trop Med Hyg 43: 650-656.
19. Oelemann WM, Teixeira MD, Veríssimo Da Costa GC, Borges-Pereira J De Castro JA, et al. (1998) Evaluation of three commercial enzyme-linked immunosorbent assays for diagnosis of Chagas' disease. J Clin Microbiol 36: 2423-2427.

20. Campos Y, Briceño L, Reina K, Figarella K, Pérez JL, et al. (2009) Serological diagnosis of Chagas disease: evaluation and characterisation of a low cost antigen with high sensitivity and specificity. Mem Inst Oswaldo Cruz 104: 914-917.

21. Flores-Chávez M, Cruz I, Rodríguez M, Nieto J, Franco E, et al. (2010) [Comparison of conventional and non-conventional serological tests for the diagnosis of imported Chagas disease in Spain]. Enferm Infecc Microbiol Clin 28: $284-293$.

22. Lorca M, Gonzalez A, Veloso C, Reyes V, Vergara U (1992) Immunodetection of antibodies in sera from symptomatic and asymptomatic Chilean Chagas' disease patients with Trypanosoma cruzi recombinant antigens. Am J Trop Med Hyg 46: 44-49.

23. Houghton RL, Benson DR, Reynolds LD, McNeill PD, Sleath PR, et al. (1999) A multi-epitope synthetic peptide and recombinant protein for the detection of antibodies to Trypanosoma cruzi in radioimmunoprecipitation-confirmed and consensus-positive sera. J Infect Dis 179: 1226-1234.

24. Ferreira AW, Belem ZR, Lemos EA, Reed SG, Campos-Neto A (2001) Enzymelinked immunosorbent assay for serological diagnosis of Chagas' disease employing a Trypanosoma cruzi recombinant antigen that consists of four different peptides. J Clin Microbiol 39: 4390-4395.

25. Maguire JH, Mott KE, Lehman JS, Hoff R, Muniz TM, et al. (1983) Relationship of electrocardiographic abnormalities and seropositivity to Trypanosoma cruzi within a rural community in northeast Brazil. Am Heart J 105: 287-294.

26. Araújo AB, Berne ME (2013) Conventional serological performance in diagnosis of Chagas' disease in southern Brazil. Braz J Infect Dis 17: 174-178.

27. Rodriguez MV, Hernandez WY, Garcia AN, Colato CM, Cardoza PG, et al (2013) ELISA seroprevalence of Trypanosoma cruzi in a cohort of heart disease patients. J Infect Dev Ctries 7: 348-354.

28. Ribeiro AL, Sabino EC, Marcolino MS, Salemi VM, Ianni BM, et al. (2013) Electrocardiographic abnormalities in Trypanosoma cruzi seropositive and seronegative former blood donors. PLoS Negl Trop Dis 7: e2078.

29. Luquetti AO (1990) Use of Trypanosoma cruzi defined proteins for diagnosis-Multicentre trial. Serological and technical aspects. Mem Inst Oswaldo Cruz 85: 497-505.

30. Umezawa ES, Bastos SF, Camargo ME, Yamauchi LM, Santos MR, et al (1999) Evaluation of recombinant antigens for serodiagnosis of Chagas' disease in South and Central America. J Clin Microbiol 37: 1554-1560. 